\title{
Usability of Spatio-Temporal Uncertainty Visualisation Methods
}

\author{
Hansi Senaratne ${ }^{1,2}$, Lydia Gerharz ${ }^{1}$, Edzer Pebesma ${ }^{1,2}$, Angela Schwering ${ }^{1}$ \\ ${ }^{1}$ Institute for Geoinformatics, University of Muenster, Germany \\ http://www.ifgi.de \\ $252^{\circ}$ North Initiative for Geospatial Open Source Software, Germany \\ http://www.52north.org
}

\begin{abstract}
The presented work helps users of spatio-temporal uncertainty visualisation methods to select suitable methods according to their data and requirements. For this purpose, an extensive web-based survey has been carried out to assess the usability of selected methods for users in different domains, such as GIS and spatial statistics. The results of the survey are used to incorporate a usability parameter in a categorisation design to characterise the uncertainty visualisation methods. This enables users to determine the uncertainty visualisation method(s) that are most suitable according to their domain of expertise. Finally, the categorisation design has been implemented and incorporated in a web-based tool as the Uncertainty Visualisation Selector. This web application can automatically recommend suitable uncertainty visualisation method(s) from user and data requirements.
\end{abstract}

Keywords: Spatio-temporal uncertainty, geovisualisation, domain-specific usability, web application

\section{Introduction}

Uncertainty visualisation aims at presenting the quantified uncertainties of data in a visual context. This is important for thorough data analysis, information derivation, as well as decision making. An example is the uncertainty visualisation of air pollutant concentration predictions across geographical regions (Van de Kassteele \& Velders 2006).

Over the past two decades, various approaches have emerged to visualise uncertainties in spatio-temporal data, and to cater different data and user requirements. These requirements can be determined from the measurement scale of the phenomenon (also referred to as data type), the data format, and the uncertainty type accounted for (Senaratne \& Gerharz 2011). User requirements refer to the usability, in particular to how easy it is to learn and understand the visualisation technique. 
Users dealing with uncertainty in data have use case specific requirements when it comes to visualising their data uncertainties (Davis \& Keller 1997). One single method cannot fulfil all requirements in order to visualise uncertain data with different combinations of the above mentioned parameters. For a user, it can be challenging to select a suitable uncertainty visualisation method for a given use case, since he/she needs to be aware of the parameters supported by the method.

Further, the various visualisation methods differ upon their usability among users of different domains. Usability is the extent to which a user can understand and utilise the functionality of a system (Nielsen 2002). This understanding comes from the experience and the background of the user. Hence, different uncertainty visualisation methods cater to different user domains. Therefore, users may find it difficult to understand and interact with specific uncertainty visualisation methods due to lack of expertise.

The objective of this paper is to help users selecting the most suitable uncertainty visualisation method(s) according to their data and user requirements. In the following, Section 2 summarises the related work. Section 3 describes a usability study on spatiotemporal uncertainty visualisations methods for different user domains. The results from the study are presented in Section 4. Section 5 introduces the Uncertainty Visualisation Selector, a tool that supports users in selecting the quantitative uncertainty visualisation method(s) upon user and data requirements. The paper finalises with a conclusion and an outlook.

\section{Background and Related Work}

\subsection{Types of Uncertainty in Spatio-Temporal Data}

Due to processing and transformations, spatio-temporal data inherits an amount of ambiguity or uncertainty (Pang 2001). Longley (2005) distinguishes three types of uncertainties for geographical data: (i) uncertainty of location data, also known as positional uncertainty, refers to not knowing the exact location of a geographic feature, such as a tree or a river course; (ii) temporal uncertainty, which is the inexactness of the temporal dimension of events that occur and vary through time, and (iii) attribute uncertainty, which refers to uncertainty of an attribute value, for example, the incompleteness of our knowledge about the temperature for a given area. In this work we focus on the third type, namely quantified uncertainties in attributes.

\subsection{Uncertainty Visualisation}

According to MacEachren (1995), vision can be considered as an information processing system, and graphics to that matter is the main language applicable to this information processing. Thus, information can be considered as the content and the graphics can be considered the container of the subjected content (Bertin 1983). Marr (1982) expressed vision as the "process of discovering from images what is present in the world, and where 
it is”. This implies that visualising uncertainties is required for comprehension. Therefore, different graphical variables have been proposed to represent spatio-temporal uncertainties.

Senaratne \& Gerharz (2011) categorised most popular spatio-temporal uncertainty visualisation methods according to the parameters, data type, data format, uncertainty type and interaction type. The data type depends on the measurement scale of an attribute and is of type continuous, ordinal or categorical (Heuvelink et al. 2007). The data format is the type of spatial data format in which the data is presented. This is typically raster or vector format. The uncertainty type of spatio-temporal data refers to the property of the data that is uncertain. This can be positional, temporal, or attribute uncertainty (Longley et al. 2005). Lastly, interaction type can be static, dynamic or interactive.

These categories are presented in Figure 1 in the form of a decision tree. By following branches of this tree for given parameter values, it is possible to choose the uncertainty visualisation method(s) according to the data and user requirements.

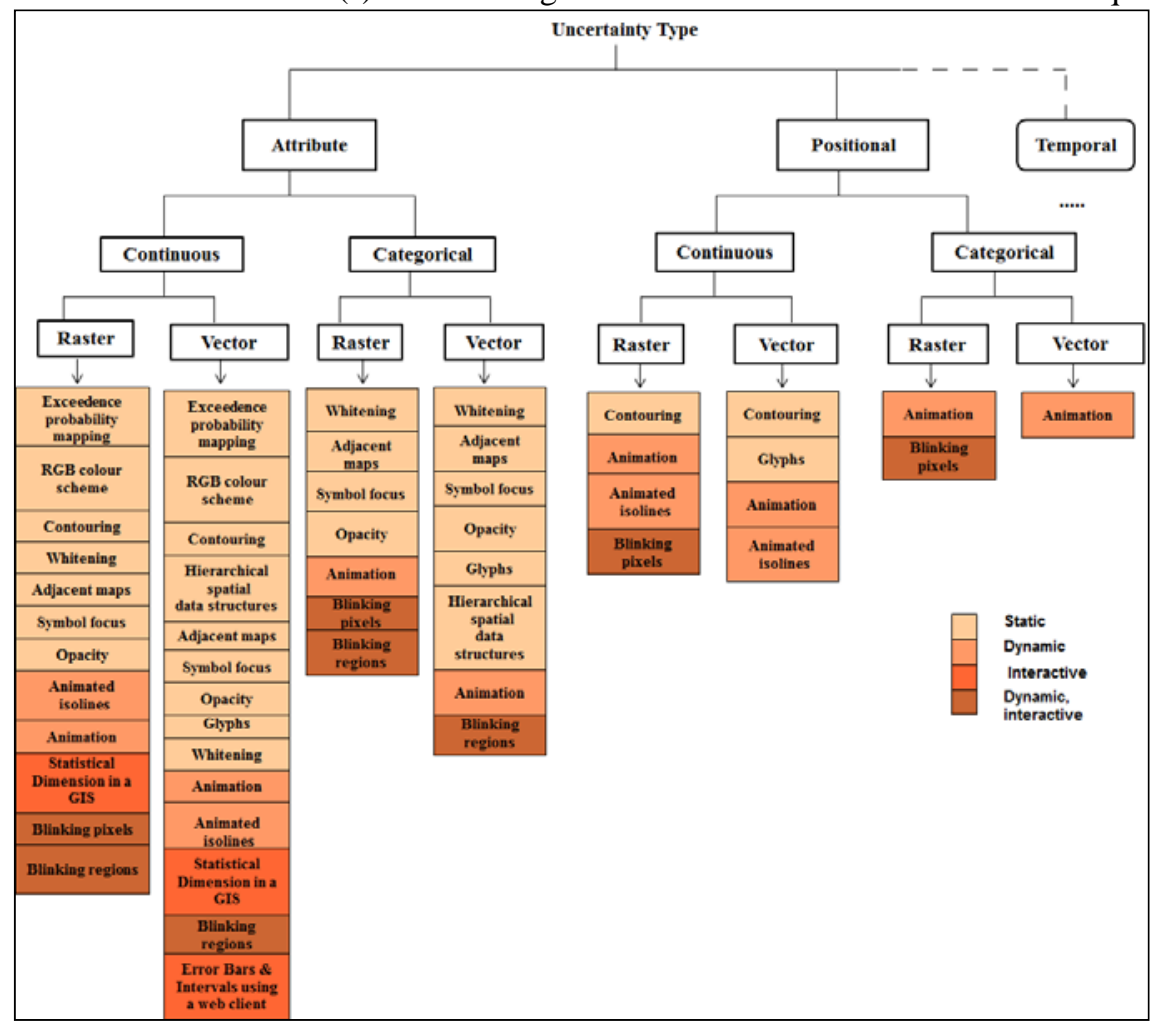

Figure 1. Categorisation of selected uncertainty visualisation methods (Senaratne \& Gerharz 2011) 


\subsection{Usability Testing}

When assessing how users interact with a system, there are particular components of usability, which can be tested. These components are as follows; easy to learn, efficient to use, easy to remember, minimal errors and subjectively pleasing.

Out of the given components of usability of a system, the easy to learn aspect also known as learnability of the selected uncertainty visualisation methods has been assessed in this research. In context of uncertainty visualisation methods, the learnability is the ability of different methods to communicate uncertainty to the users.

From a study that was done on usability testing by Nielsen (1989), it was evident that four out of the ten major usability effects were due to the individual differences between users and only two were due to the differences of the given tasks. This proves the importance of acquainting the user and treating this aspect as an important component when testing the usability of a system. Usability of the different uncertainty visualisation methods depends on the user's background experiences (Gerharz \& Pebesma 2009). Therefore, this research aims at assessing which uncertainty visualisation methods are most suitable for different user domains, e.g. statistics or urban planning.

\subsection{Usability Studies on Uncertainty Visualisation Methods}

Since the early 1990s, usability tests were conducted in numerous styles giving importance to one or more components of usability, achieving interesting and influential results.

Evans (1997) assessed Static Colour Bivariate Maps (MacEachren et al. 2005), Flickering Animation method (Fisher 1993) and Toggling (MacEachren 1992), out of which the Toggling method was least preferred by all users.

MacEachren et al. (1998) assessed Adjacent Maps (MacEachren et al. 1998), Texture Overlay method (Kardos et al. 2003) and a Colour Model (MacEachren et al. 2005). The results enlightened that users preferred the Texture Overlay method for exploratory purposes and the Colour Model for presentation purposes.

Cliburn et al. (2002) evaluated the usability of a Colour Model (Hengl et al. 2002), Transparency method (MacEachren et al. 2005) and Glyphs method (Pang 2001). Users with scientific backgrounds preferred the use of Glyphs, users with less experience in sciences preferred the Colour Model, and Transparency method was preferred by decision makers.

Aerts et al. (2003) assessed the Adjacent Maps method (MacEachren et al. 1998) and the Animated Toggling method (MacEachren 1992) where, users unanimously preferred Adjacent Maps slightly over the Toggling method.

More recently, Gerharz \& Pebesma (2009) assessed Adjacent Maps (MacEachren et al. 1998), Whitening (Hengl 2003) and Aguila (a tool that introduces a statistical dimension to GIS) (Pebesma et al. 2007). Adjacent Maps method was preferred by all users and the interactive method implemented in Aguila was found to be more suitable as an expert tool. 


\section{Usability Study}

To test the user-specific requirements, the participants were asked to select their domain of expertise out of map visualisation, urban planning, decision support, GIS, and statistics domains. In the following section, an overview of the data sets is given before the created uncertainty visualisations are described in Section 3.2. In Section 3.3, the design of the Web-based study is explained.

\subsection{Data Sets}

The first data set was a residual Kriging analysis of PM10 concentration data in raster format with $25 \mathrm{~km}$ x $25 \mathrm{~km}$ cell size. PM10 are airborne particles with $10 \mu \mathrm{m}$ diameters or less. The Kriging interpolation was performed on annual averages of PM10 measurements over Europe for the year 2005 from the AirBase database provided by the EEA $^{1}$ (European Environmental Agency). The uncertainties of these PM10 maps are in essence Kriging variances (Gerharz \& Pebesma 2009). Resulting maps represent attribute uncertainties for continuous data in raster format.

The second data set was acquired by the Global Land Cover-mapping $2000^{2}$ project and contained land use data for Asia acquired by the VEGETATION instrument on board of the SPOT 4 with a spatial resolution of $1 \mathrm{~km}$ and a temporal resolution of 1 day (Agrawal et al. 2003). The uncertainty of these land use classes were caused by wrong labelling, missing classes, wrong position of the boundaries between classes and disappearances of small patches of land. These uncertainties were validated through a confidence building method where captured data was compared with ancillary data and through sampling methods. The land use data hold attribute uncertainties for categorical data in vector format.

The third data set comprised of simulated ground level Ozone data. This data was encoded in conformance to the Observations and Measurements standard ${ }^{3}$ of the Open Geospatial Consortium. These ground level Ozone data included attribute uncertainties for continuous data in vector format.

\subsection{Creation of Uncertainty Visualisation Methods}

The uncertainty visualisation methods subjected in the usability assessment were: Adjacent Maps, Contouring, Symbols, Error Bars and Confidence Intervals, and Aguila. The selection was based on the needs of the EU FP-7 project UncertWeb ${ }^{4}$. The methods

\footnotetext{
${ }^{1}$ http://www.eea.europa.eu/themes/air/airbase

${ }^{2}$ http://bioval.jrc.ec.europa.eu/products/glc2000/products.php

${ }^{3}$ http://www.opengeospatial.org/standards/om

${ }^{4}$ http://www.uncertweb.org/
} 
were carefully inspected for their accordance with the categorisation to ensure that the provided data, in its nature were compatible to be used with the methods. In the following sections, the visualisations created according to those methods are described.

\subsubsection{Adjacent Maps Visualisation}

The adjacent maps of two side by side raster maps were created using ArcGIS 10.0 (Ormsby et al. 2010) (Figure 2), to visualise the value and the associated uncertainty within the PM10 data set side by side. In order to create maps with colour sequences which make sense to the cognitive perception, the suggested colour palettes from ColorBrewer (Brewer et al. 2003) were used, where these colour palettes are formalised according to the nature of data. The selected colour schemes could also be identified without any confusion due to red-green colour blindness.

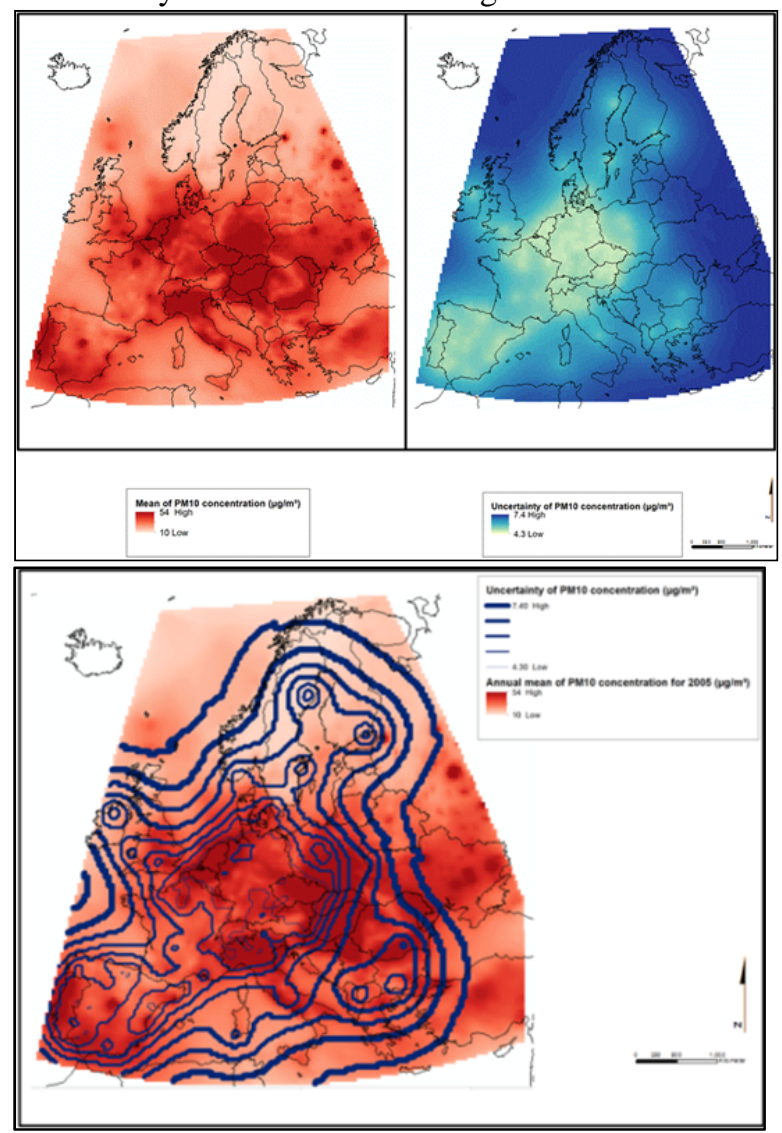

Figure 2. (Top) Adjacent Maps method. PM10 concentration data (left) and uncertainty of the PM10 data (right) over Europe are represented on two side by side maps

Figure 3. (Bottom) Contouring method. PM10 concentration data is presented in the background and uncertainty in the data is represented through contours in the foreground 


\subsubsection{Contouring Visualisation}

The Contouring method (Dutton 1992) displays contour lines that connect locations where the attribute has a constant value (Longley et al. 2005). In Figure 3, this technique was applied to depict the uncertainties of the PM10 concentration over Europe. In the background, PM10 concentration data is depicted as raster through colour saturation. Uncertainty of PM10 concentration shown in the foreground is represented through thickening contours. These contours were created in ArcGIS 10.0 using the 3D Analyst tool.

\subsubsection{Symbols Visualisation}

The Symbols method (Pang 2001) expresses the figurative similarities of objects based on shape or colour (Bertin 1983). Assigning colours to symbols was done with much caution as it needs to convey a realistic meaning to the users such that they can relate to it. Here, the uncertainties in the land use data set of Asia were depicted using circular symbols as seen in the foreground of Figure 4. Different land use classes over Asia are displayed in the background. The increasing uncertainty was shown by symbols of increasing size and varying colour. The colours green, yellow, orange and red were used in order, to represent increasing uncertainties, red communicating highest uncertainties.

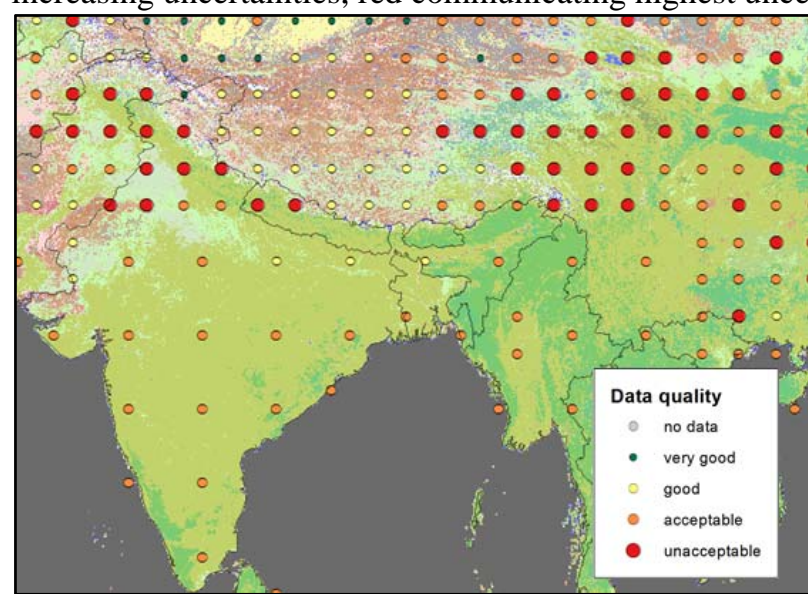

Figure 4. Uncertainty of land use classes represented through symbols of varying colour and size 


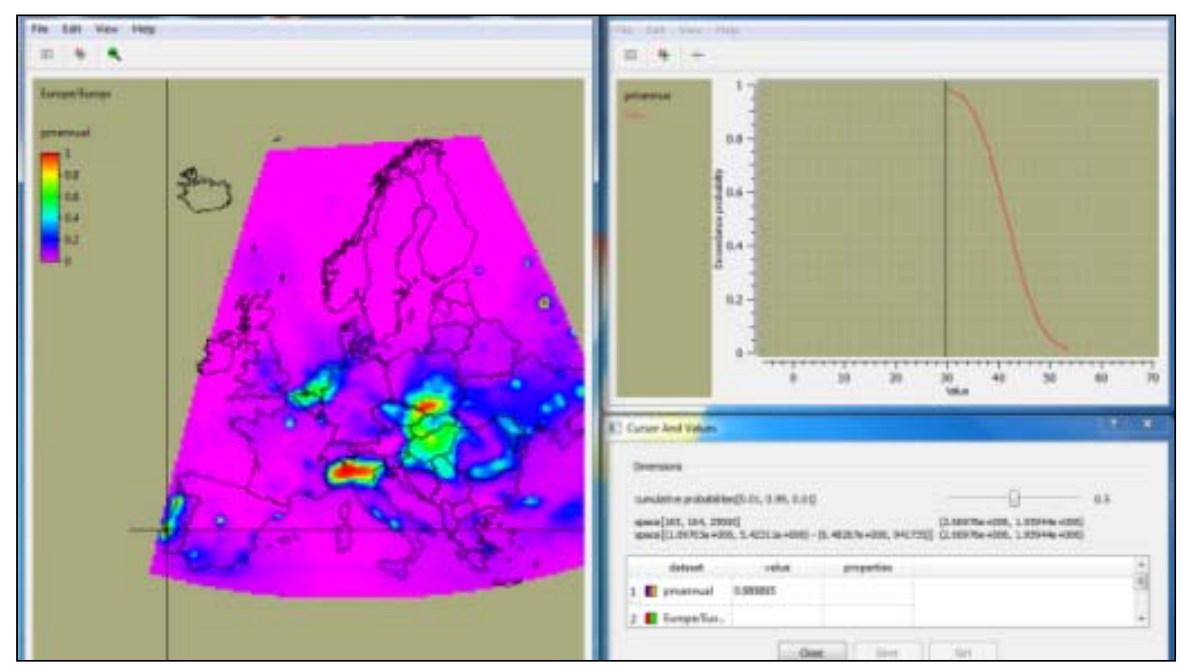

Figure 5. Statistical dimension in a GIS using Aguila

\subsubsection{Visualisation of spatio-temporal probability distributions using Aguila}

Aguila is an open source, statistical, interactive visualisation tool for analysing spatiotemporal data that has uncertainty data encoded as probability distributions (Pebesma et al. 2007). The annual mean PM10 concentration data was represented on a map in a separate window where different values were represented through a colour ramp (Figure 5, left window). Each raster cell shows a characteristic of the full probability density function (PDF) for this cell, estimated from PM10 mean and variance, under the assumption that the distribution is normal. Figure 5, upper right window, shows the probability of exceedance for the grid cell under the cross hair of the left window map. The cursor in the PDF window can be used to define which quantile is shown in the map. Alternatively, the cursor can be used to select values and visualise the cumulative or exceedance probability according to that value (see Figure 5). The cursor on the figure is pointed at Southern Portugal and the corresponding exceedance probability for threshold $30 \mu \mathrm{g} / \mathrm{m}^{3}$ is shown in the PDF graph in top right window, the value being nearly 1 . Exceedance probability is the probability that a quantity (here: PM10) exceeds a threshold value. From this figure, it is evident that the probability of exceeding the threshold is quite high, implying that the uncertainty about exceeding this threshold is low. The window below the PDF graph in Figure 5 represents the respective cursor values for the probability.

Because it takes some time and skills to operate Aguila, for our survey, to assess the usability of this method, a video clip was recorded for a given scenario depicting the exceedance probability for certain regions over Europe. Thus, even though this method is dynamic and interactive in nature, only the dynamic aspect of it could be tested through the video clip. 


\subsubsection{Error Bars and Intervals Visualisation}

Error bars (Olston \& Mackinlay 2002) are a popular means to represent the distribution of data values which causes uncertainty. Figure 6 shows the Web client developed in the UncertWeb project that has been used to create visualisations for the ground level Ozone data set. As shown in the figure, the visualisation includes on the left a time series for the ground level Ozone data at one location with the error bars at each data point to illustrate the amount of uncertainty involved in the measurements. The probability coverage of the error bars can be adjusted by the slider on the right side of the time series plot. For each time step the full PDF for the Ozone values at this point can be shown.

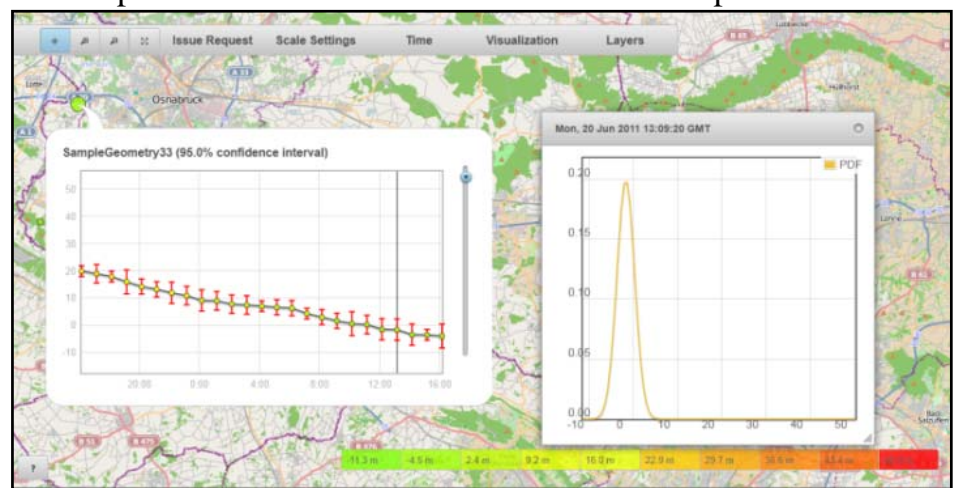

Figure 6. Uncertainty of ground level Ozone depicted through Error bars and its PDF at a given time

Similarly, intervals can be used in an identical manner. This visualisation method was developed through an Open Layers Web client. A video clip that depicts an interaction with this client was used in the usability study to present the method to the participants.

\subsection{Design of the Usability Study}

Designing an approach similar to Evans (1997), the usability study was sectioned into three stages. At the first stage it was assessed if the user acknowledges spatio-temporal uncertainty when presented visually. The second stage of the study involved decision making questions where the participants were presented with the visualisation and had to cautiously analyse the data and uncertainty together in order to answer the questions. It was envisioned that the participants apply the knowledge acquired from the first stage of the study, when making the decisions.

After acquiring an idea of the different uncertainty visualisation methods and decision making problems, in the third stage users had to mark their preferred uncertainty visualisation methods. Furthermore, through the correspondence between the performances (statistics of correct and wrong answers) and preferences, the learnability aspect of uncertainty visualisation methods was assessed. In order to assess the feasibility of the research design of the web-based survey, a pilot study was initially carried out. 
The web-based usability study ${ }^{5}$ was created using the LimeSurvey online tool ${ }^{6}$. The first set of questions acquired personal information of the participant that was not intended to trace back to the user. They comprised of the age, gender and the background experience information. Following the strategy used by Aerts et al. 2003, the participants were given the choice of five different domains to mark as background experience. These domains were, map visualisation, urban planning, decision support, GIS and statistics. Participants who marked one or more of these domains were considered as experienced in dealing with uncertainty in their data. The participants who did not belong to any of the five domains were given the option of marking "I don't have experience in any of the above fields” (as done by Aerts et al. 2003). Additionally, a text field was provided where the participant could type in their domain of expertise, if they did not belong to any of the explicitly stated backgrounds. When evaluating, this field was carefully analysed not to miss out any participant in categorising them into one of the five domains. Through this user categorisation it was possible to evaluate the results of the survey with respect to the different domains, and hence conclude the most suitable uncertainty visualisation method(s) for each domain user group.

\section{Results and Discussion of the Usability Survey}

This section provides an analysis of the Web-based usability survey responses. The survey was forwarded to several mailing lists and networks in order to acquire a substantial amount of responses for a fine evaluation. This resulted in a total of 140 responses out of which 81 participants completed the survey, resulting in a 58\% success rate. Only the fully completed surveys were included in the analysis. Out of the 81 participants, the frequency of users in GIS domain was 66 (31.7\%), map visualisation was 52 (25\%), statistics was 47 (22.6\%), decision support was 21 (10.1), urban planning was 15 (7.2) and the frequency of users in the "Other" category was 7 (3.4\%). The users in the "Other" category were the users who did not belong to any of the domains. The analysis was performed for three main aspects: Section 4.1 analyses the performance of the participants, while Section 4.2 evaluates based on the users responses, which methods were preferred in the end. Section 4.3 carries out a correspondence analysis between performance and preference.

\subsection{User Performance}

Performance within the context of the usability assessment in this research is the proportion of correct answers given by the participant to the decision making questions that were posed at the second stage of the survey. The understanding that was gained at

\footnotetext{
${ }^{5}$ http://surveys.ifgi.de/index.php?sid=47734\&lang=en

${ }^{6}$ http://www.limesurvey.org/
} 
each user domain was evaluated through the percentage of users that answered correctly for the question of each method as shown in Figure 7.

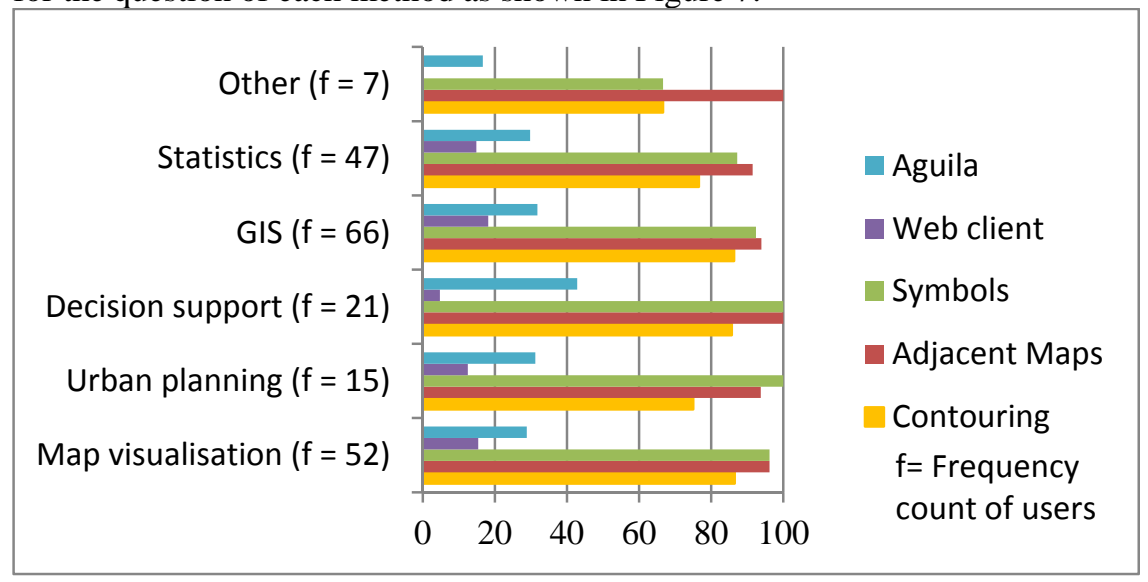

Figure 7 Percentage of users from each domain that answered correctly for each method

A majority of the users from the five domains answered correctly for the Adjacent Maps and Symbols methods, with the method Contouring following shortly behind. The Web client and the Aguila methods were the most difficult to answer. This difficulty can be reasoned from the comments that were given by some participants when they were asked about the comprehensibility of the method, as the lack of descriptiveness of the graphs in the Web client and in Aguila.

Further, representing these two interactive methods as a dynamic video probably accounted for the poor performance as well. As the users did not get to interact with these two methods, it clearly prevented them from exploring the tools and further advancing their knowledge. A majority of users from the "Other" category performed well in the Adjacent Maps method with Symbols and Contouring following behind. As of the domain users, these participants also found it difficult to understand the Web client and Aguila methods, which might explain their poor performance.

\subsection{User Preferences}

At the very end of the survey the users were asked which method(s) they would choose if they had to visualise their data uncertainties, based on visual appeal and comprehensibility (Figure 8). Since the users were given the option of marking one or more methods, a majority of the users from the different domains opted Adjacent Maps as their second choice along with others. Though not as significant as for the Adjacent Maps method, there was also a slight popularity for the Symbols method. However the methods Contouring, Aguila and the Web client remain dominant as a majority choice. Contrastingly, the "Other” users, picked Adjacent Maps and Aguila methods as a majority preference. 


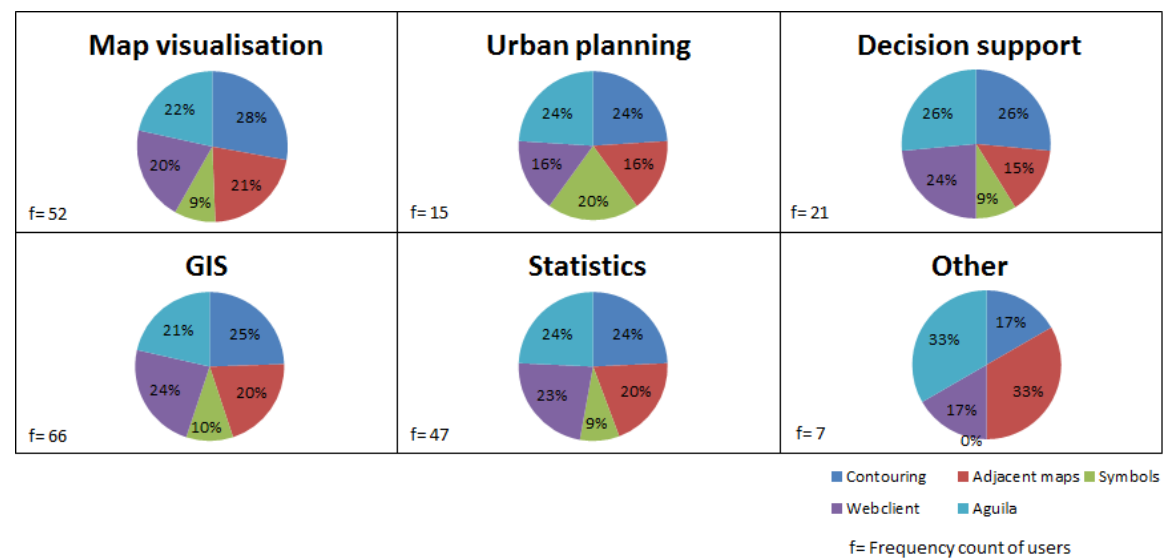

Figure 8. Summary of results when the users were asked which uncertainty visualisation method(s) they would use if they had to visualise their uncertain data

\subsection{Correspondence between User Performance and User Preference}

Two way tables (Tables 1-6) containing measures of correspondence between the user's performance and preference have been analysed for each user domain. This was used to analyse which uncertainty visualisation method(s) are most suitable to be used by a respective user group. These two factors are important in determining the most suitable methods for a user group, as performance conveys what the user understood the best and preference conveys what the users desires most to visualise their data uncertainties. Therefore, the highest correspondence between these two factors reveals the most suitable uncertainty visualisation method(s) for the different user domains.

Since the users were allowed to select multiple preferences, the totals of each column and row do not indicate the total number of users belonging to that respective domain. The highlighted cells in these tables merely depict how many users performed well at each method and in turn selected that particular method as the preferred method to use.

Table 1. Correspondence between performance and preference for Map Visualisation domain

\begin{tabular}{|c|c|c|c|c|c|c|}
\hline & & & Preference & & & \\
\hline \multirow{5}{*}{ 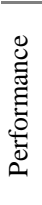 } & Contouring & $\begin{array}{l}\text { Contouring } \\
\mathbf{1 7}\end{array}$ & $\begin{array}{l}\text { Adj. Maps } \\
17\end{array}$ & $\begin{array}{l}\text { Symbols } \\
5\end{array}$ & $\begin{array}{l}\text { WebClient } \\
16\end{array}$ & $\begin{array}{l}\text { Aguila } \\
15\end{array}$ \\
\hline & Adj. Maps & 21 & 16 & 7 & 15 & 16 \\
\hline & Symbols & 20 & 17 & 7 & 15 & 15 \\
\hline & WebClient & 4 & 2 & 0 & 2 & 2 \\
\hline & Aguila & 5 & 4 & 2 & 3 & 6 \\
\hline
\end{tabular}


Table 2. Correspondence between performance and preference for Urban Planning domain

\begin{tabular}{|c|c|c|c|c|c|c|}
\hline \multirow{7}{*}{ 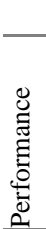 } & & \multicolumn{3}{|c|}{ Preference } & \multirow[b]{2}{*}{ WebClient } & \multirow[b]{2}{*}{ Aguila } \\
\hline & & Contouring & Adj. Maps & Symbols & & \\
\hline & Contouring & 4 & 3 & 3 & 4 & 5 \\
\hline & Adj. Maps & 5 & 3 & 4 & 4 & 6 \\
\hline & Symbols & 5 & 3 & 4 & 4 & 6 \\
\hline & WebClient & 1 & 0 & 0 & $\mathbf{0}$ & 1 \\
\hline & Aguila & 1 & 1 & 1 & 0 & 2 \\
\hline
\end{tabular}

Table3. Correspondence between performance and preference for Decision Support domain

\begin{tabular}{|c|c|c|c|c|c|c|}
\hline & & & Preference & & & \\
\hline \multirow{6}{*}{ 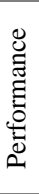 } & & Contouring & Adj. Maps & Symbols & WebClient & Aguila \\
\hline & Contouring & 8 & 5 & 2 & 8 & 8 \\
\hline & Adj. Maps & 9 & 5 & 3 & 8 & 9 \\
\hline & Symbols & 9 & 5 & 3 & 8 & 9 \\
\hline & WebClient & 0 & 0 & 0 & $\mathbf{0}$ & 1 \\
\hline & Aguila & 5 & 2 & 0 & 1 & 4 \\
\hline
\end{tabular}

Table 4. Correspondence between performance and preference for GIS domain

\begin{tabular}{|c|c|c|c|c|c|c|}
\hline & & & Preference & & & \\
\hline \multirow{6}{*}{ 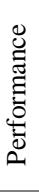 } & & Contouring & Adj. Maps & Symbols & WebClient & Aguila \\
\hline & Contouring & 19 & 18 & 7 & 22 & 19 \\
\hline & Adj. Maps & 23 & 18 & 10 & 20 & 21 \\
\hline & Symbols & 23 & 19 & 10 & 21 & 19 \\
\hline & WebClient & 4 & 4 & 1 & 2 & 4 \\
\hline & Aguila & 4 & 4 & 3 & 6 & 8 \\
\hline
\end{tabular}

Table 5. Correspondence between performance and preference for Statistics domain

\begin{tabular}{|c|c|c|c|c|c|c|}
\hline & & & Preference & & & \\
\hline \multirow{6}{*}{ 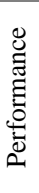 } & & Contouring & Adj. Maps & Symbols & WebClient & Aguila \\
\hline & Contouring & 14 & 10 & 4 & 15 & 13 \\
\hline & Adj. Maps & 16 & 12 & 6 & 14 & 17 \\
\hline & Symbols & 16 & 12 & 5 & 14 & 14 \\
\hline & WebClient & 3 & 2 & 0 & 1 & 2 \\
\hline & Aguila & 4 & 4 & 1 & 5 & 5 \\
\hline
\end{tabular}

Table 6. Correspondence between performance and preference for "Other" users

\begin{tabular}{|c|c|c|c|c|c|c|}
\hline & & & Preference & & & \\
\hline \multirow{6}{*}{ 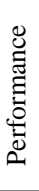 } & & Contouring & Adj. Maps & Symbols & WebClient & Aguila \\
\hline & Contouring & 1 & 2 & 0 & 1 & 0 \\
\hline & Adj. Maps & 1 & 2 & 0 & 1 & 2 \\
\hline & Symbols & 2 & 2 & 1 & 1 & 1 \\
\hline & WebClient & 0 & 0 & 0 & $\mathbf{0}$ & 0 \\
\hline & Aguila & 0 & 1 & 0 & 0 & $\mathbf{0}$ \\
\hline
\end{tabular}

In Tables 1 to 6 it is evident that the correspondence between performance and preference dominates in static visualisation methods (Contouring, Adjacent Maps and Symbols) when compared to the two dynamic visualisation methods.

Regardless of the poor performances in the Web client and Aguila methods, there is a substantial number of participants who gave their preference for these two methods. This 
can be explained by the misinterpretations of the two methods and hence the possible impression of the participants that they answered correctly. As stated before, this is possibly a result of representing the interactive methods as a dynamic video.

In conclusion of the above described evaluation, the most suitable spatio-temporal uncertainty visualisation methods are derived for each user domain. The method(s) with highest correspondence are the most suitable for that particular user domain. These rankings are presented in Table 7.

Table 7. Most suitable uncertainty visualisation methods; left to right with decreasing suitability

\begin{tabular}{ll}
\hline Domain & Most Suitable Uncertainty Visualisation Method(s). \\
\hline Map Visualisation & Contouring, Adjacent Maps, Aguila, Web client \\
Urban Planning & Contouring, Symbols, Aguila, Web client \\
Decision Support & Contouring, Aguila, Web client \\
GIS & Contouring, Adjacent Maps, Aguila, Web client \\
Statistics & Contouring, Adjacent Maps, Aguila, Web client \\
Other & Adjacent Maps \\
\hline
\end{tabular}

\section{The Uncertainty Visualisation Selector}

This section presents the design of the categorisation of spatio-temporal uncertainty visualisation methods and the implementation of the Uncertainty Visualisation Selector tool $^{7}$. Upon specification of user and data requirements, the tool automatically selects an uncertainty visualisation method(s) using the decision tree derived from the categorisation. Figure 9 shows the UML class diagram, which gives an overview of the structure of the Uncertainty Visualisation Selector. The abstract type visualisation method in the centre is sub-classed into three main categories based on the interaction type, namely static, dynamic and interactive visualisation methods. The other parameters which are discussed above (uncertainty type, data type and data format) are as well modelled as separate abstract types and aggregated by the visualisation method type. Concrete parameter values (e.g. positional uncertainty as a specific uncertainty type) are modelled as subtypes of the parameter types.

To introduce the usability aspect, the visualisation method is aggregated by the type Domain which is sub-classed into the considered types of domains (e.g. GIS, Statistics, etc.). Each concrete domain has an ordered list of suitable uncertainty visualisation methods as derived from the usability study.

This categorisation design was implemented in Java and integrated into a web application as a JavaServer Page (JSP) (Falkner et al. 2001). The web application provides dropdown menus from which users can select the different parameters. After clicking the submit button, a list of uncertainty visualisation methods are presented, that suffice the specified requirements and that were derived as most suitable to the user's domain, based on the usability study. However, only a limited number of uncertainty visualisation methods are assessed on their usability. Therefore, in case the specified combination of parameters does not confirm to any of the assessed methods, the user is prompted to select "None"

${ }^{7}$ http://geoviqua.dev.52north.org/UVS/ 
from the domain list and submit again to retrieve a list of visualisation methods without considering the aspect of domain suitability.

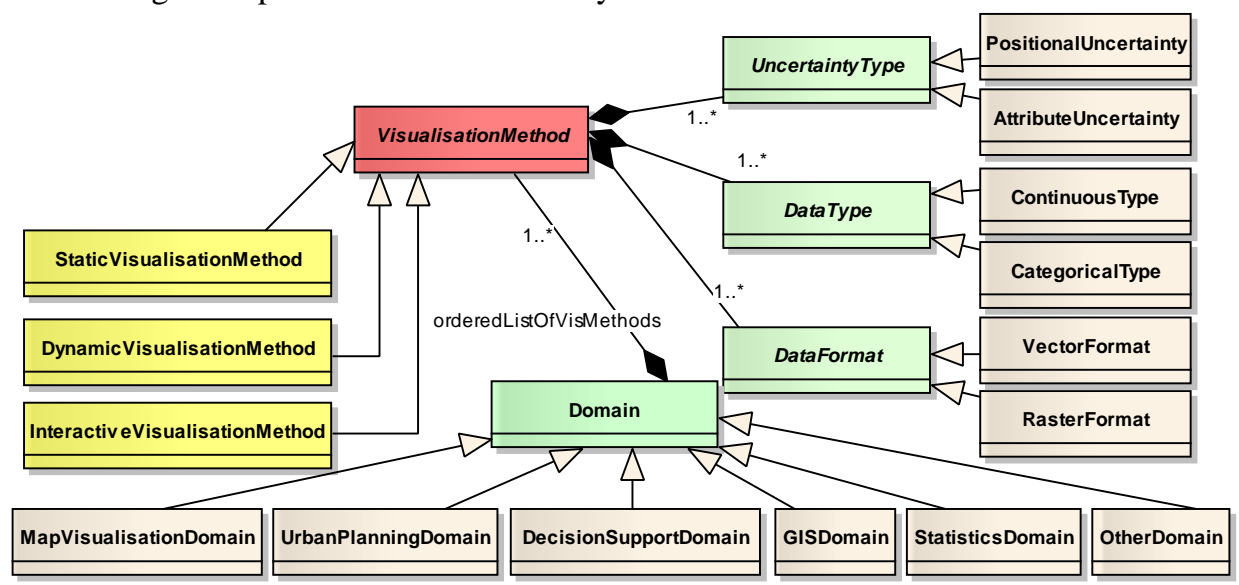

Figure 9. UML class diagram depicting the interrelation between uncertainty visualisation methods and the parameters that are used to characterise these methods

\section{Conclusion \& Outlook}

This research helps users to select uncertainty visualisation methods. We realised that, even if the users are provided with a set of methods to use, from a categorisation based upon their data type, uncertainty type, data format and preferred interaction type, these methods were not necessarily usable by all kinds of users. Building upon this, a webbased usability study was conducted on selected uncertainty visualisation methods. The participants who took part in this study categorised themselves into the user domains: map visualisation, urban planning, decision support, GIS and statistics. Through a measurement of correspondence between their performance and preference, the most suitable uncertainty visualisation method(s) were derived for each user domain (Table 7). The user preference was based on the visual appeal and comprehensibility of the methods. Subsequently, the categorisation of spatio-temporal uncertainty visualisation methods was implemented in a web application, the Uncertainty Visualisation Selector. User domain was added as a parameter in order to further characterise the methods. The Uncertainty Visualisation Selector enables the user to specify the data requirements (data type, data format and uncertainty type) and user requirements (domain he/she belongs to) upon which, suitable uncertainty visualisation methods are generated through realising the categorisation.

The design of the usability study is described here as a transparent and generic approach which can be followed to assess the usability of other methods in future. Compared to related studies, this usability study with 81 participants was comparatively large (to the best knowledge of the authors, only the study by MacEachren et al. (1998) had a larger number, consisting of 84 participants). Instead of the common distinction of expert users and non-expert users, a more fine grain categorisation between users according to their 
domains of expertise had been applied. Hence, the suitable uncertainty visualisation method(s) could be derived for each domain.

However, by categorising the participants of the survey into different domains, the conducted usability study lacked inexperienced users which resulted in a weak evaluation for this group. In future usability studies of this nature it seems useful to allot more inexperienced participants in order to derive a stronger evaluation for this group.

A limiting factor was the representation of the two interactive methods (Web client and Aguila method) in the form of dynamic videos as part of the web-based survey. In future, it is commended to assess the usability on these two methods in their interactive nature, in order to get an enhanced evaluation of their usability.

Looking at the categorisation of spatio-temporal uncertainty visualisation methods, additional parameters could be added in future. One such parameter is the uncertainty representation type of the uncertain data that needs to be visualised. Not all methods can deal with the different uncertainty representation types (full PDFs, quantiles). Therefore, by including such a parameter, the purpose of the web application would be further enhanced. This web application has the potential to be integrated with a visualisation tool to also create visualisations.

\section{Acknowledgement}

This work was supported in part by the European Commission through the FP7 research projects "Quality aware Visualisation for the Global Earth Observation System of Systems (GeoViQua)" (FP7 ENV 2010-1-265178) and "Uncertainty enabled Model Web (UncertWeb)" (FP7/2007-2013).

\section{References}

Aerts JCJH, Clarke KC, Keuper AD. (2003). Testing Popular Visualization Techniques for Representing Model Uncertainty. Cartography and Geographic Information Science, 30(3), 249-261.

Agrawal S, Joshi PK, Shukla Y, Roy PS. (2003). SPOT Vegetation Multi Temporal Data for Classifying Vegetation in South Central Asia. Current Science, 84(11), 1440-1448.

Bertin J. (1983). Semiology of Graphics: Diagrams, Networks, Maps. (Translation by William J. Berg). The University of Wisconsin Press, Madison, WI, USA.

Brewer CA, Hatchard GW, Harrower MA. (2003). ColorBrewer in Print: A Catalog of Color Schemes for Maps. Catography and Geographic Information Science, 30(1), 5-32.

Cliburn DC, Fedemma JJ, Miller JR, Slocum TA. (2002). Design and Evaluation of a Decision Support System in a Water Balance Application. Computer \& Graphics, 26, 931-949.

Davis TJ, Keller CP. (1997). Modelling and Visualizing Multiple Spatial Uncertainties. Computers \& Geosciences, 23(4), 397-408. 
Dutton G. (1992). Handling Positional Uncertainty in Spatial Databases. In: Proceedings of the 5th International Symposium on Spatial Data Handling, Charleston, SC, USA. August 1992. 460469.

Evans BJ. (1997). Dynamic Display of Spatial Data Reliability: Does it Benefit the Map User? Computers \& Geosciences, 23, 409-422.

Falkner J, Timney J, GalbraithI B. (2001). Beginning JSP Web Development. Wrox Press Ltd. Birmingham, UK.

Fisher P. (1993). Visualizing Uncertainty in Soil Maps by Animation. Cartographica, 30(2+3), 2027.

Gerharz LE, Pebesma EJ. (2009). Usability of Interactive and Non-Interactive Visualisation of Uncertain Geospatial Information. In: Reinhardt, W., Krüger, A., Ehlers, M. (Eds.), Geoinformatik 2009 Konferenzband, Osnabrück, Germany. 31 March-1 April 2009. 223-230.

Hengl T, Walvoort DJJ, Brown A. (2002). Pixel (PM) and Colour Mixture (CM): GIS Techniques for Visualisation of Fuzziness and Uncertainty of Natural Resource Inventories. In: Hunter, G. J., Lowell, K. (Eds.), Proceedings of the 5th International Symposium on Spatial Accuracy Assessment in Natural Resources and Environmental Sciences (Accuracy 2002), Melbourne, Australia. 300-309.

Hengl T. (2003). Visualisation of Uncertainty Using the HIS Colour Model: Computations with colours. In: Proceedings of the 7th International Conference on GeoComputation, Southampton, UK.8-10 September 2003. 8-17.

Heuvelink GBM, Brown JD, van Loon EE. (2007). A Probabilistic Framework for Representing and Simulating Uncertain Environmental Variables. International Journal of Geographical Information Science, 21(5), 497-513.

Kardos JD, Moore A, Benwell GL. (2003). Visualising Uncertainty in Spatially-Referenced Attribute Data Using Hierarchical Spatial Data Structures. In: Proceedings of the 7th International Conference on GeoComputation, Southhampton, UK. 8-10 September 2003.

Longley PA, Goodchild MF, Maguire DJ, Rhind DW. (2005). Geographic Information Systems and Science (2nd edition). Wiley \& Sons. West Sussex, UK.

MacEachren AM. (1992). Visualising Uncertain Information. Cartographic Perspective, 13, 10-19.

MacEachren AM. (1995). How Maps Work: Representation, Visualization and Design. Guilford Press. New York, USA.

MacEachren AM, Brewer CA, Pickle IW. (1998). Visualising Georeferenced Data: Representing Reliability of Health Statistics. Environment and Planning A, 30, 1547-1561.

MacEachren AM, Robinson A, Hopper S, Gardner S, Murray R, Gahegan M, Hetzler E. (2005). Visualizing Geospatial Information Uncertainty: What We Know and What We Need to Know. Cartography and Geographic Information Science, 32, 139-160. 
Marr D. (1982). Vision. W.H. Freeman and Company. New York, USA.

Nielsen J. (1989). The Matters That Really Matter for Hypertext Usability. Proceedings of Hypertext 89. ACM Press. New York, USA. 239-248.

Nielsen J. (2002). The Usability Engineering Life Cycle. Computer, 25 (3), 12-22.

Olston C, Mackinlay J. (2002). Visualizing Data with Bounded Uncertainty. In: Proceedings of the IEEE Symposium on Information Visualization, Boston, MA, USA. 28-29 October 2002. 3740.

Ormsby T, Napoleon EJ, Burke R, Groessl C, Bowden L. (2010). Getting to Know ArcGIS Desktop (2nd edition). Esri Press. Redlands, USA.

Pang AT. (2001). Visualizing Uncertainty in Geo-spatial Data. In: Proceedings of the Workshop on the Intersections between Geospatial Information and Information Technology, Arlington, TX, USA. October 2001.

Pebesma EJ, de Jong K, Briggs D. (2007). Interactive Visualization of Uncertain Spatial and Spatiotemporal Data under Different Scenarios: An Air Quality Example. International Journal of Geographical Information Science, 21(5), 515-527.

Senaratne H, Gerharz L. (2011). An Assessment and Categorisation of Quantitative Uncertainty Visualisation Methods. The 14th AGILE International Conference on Geographic Information Science. 18.-21 April, Utrecht, Netherlands. Online available: http://plone.itc.nl/agile_old/Conference/2011-utrecht/contents/pdf/posters/p_144.pdf. Accessed on 20 Sep 2011.

Van de Kassteele J, Velders GJM. (2006). Uncertainty Assessment of Local NO2 Concentrations Derived from Error-in-Variable External Drift Kriging and its Relationship to the 2010 Air Quality Standard. Atmospheric Environment, 40(14), 2583-2595. 